\title{
Gastric cancer is highly prevalent in Lynch syndrome patients with atrophic gastritis
}

\author{
Hourin Cho ${ }^{1} \cdot$ Masayoshi Yamada ${ }^{1}\left(\mathbb{D} \cdot\right.$ Shigeki Sekine $^{2} \cdot$ Noriko Tanabe $^{3} \cdot$ Mineko Ushiama $^{3,4} \cdot$ Makoto Hirata $^{3}$. \\ Gakuto Ogawa ${ }^{5}$. Masahiro Gotoh ${ }^{4} \cdot$ Teruhiko Yoshida $^{3} \cdot$ Takaki Yoshikawa $^{6}$ - Yutaka Saito ${ }^{1}$. Aya Kuchiba ${ }^{5}$. \\ Ichiro Oda ${ }^{1} \cdot$ Kokichi Sugano ${ }^{3,7}$
}

Received: 15 May 2020 / Accepted: 4 August 2020 / Published online: 13 August 2020

(c) The International Gastric Cancer Association and The Japanese Gastric Cancer Association 2020

\begin{abstract}
Background Although gastric cancer is one of the Lynch syndrome (LS)-related tumors, the clinicopathological features of gastric cancer in patients with LS remain uncertain. To investigate the incidence risk and clinicopathological features of gastric neoplasms in LS, we conducted a retrospective cohort study in Japanese LS patients.

Methods LS patients with pathogenic mismatch repair (MMR) gene variants were extracted from the LS registry of the National Cancer Center Hospital, Japan. Cumulative risks of gastric neoplasm, including dysplasia and cancer, were estimated using the Kaplan-Meier method. Gastric atrophy was evaluated endoscopically and/or histologically. Immunohistochemical staining for MMR proteins was performed for all available specimens.

Results Of 118 eligible patients, 26 patients were diagnosed with 58 gastric neoplasms. The cumulative incidence of gastric neoplasm was $41.0 \%$ (95\% confidence interval, 26.9-55.0) at the age of 70. Of these, 13 (50\%) patients developed synchronous and/or metachronous multiple gastric neoplasms. Among the 49 gastric neoplasms available for detailed pathological evaluation, all were associated with intestinal metaplasia. Immunohistochemically, 42 (86\%) were MMR-deficient. The individuals with gastric atrophy had a significantly higher risk of developing gastric neoplasms compared with those without gastric atrophy (26 cases/54 individuals vs. 0 cases/53 individuals) $(P=0.026)$.

Conclusion LS patients, particularly those with atrophic gastritis, are at high risk of gastric neoplasm and often develop multiple tumors. Endoscopic surveillance for gastric cancer is recommended for LS patients, especially those with atrophic gastritis.
\end{abstract}

Keywords Stomach neoplasms $\cdot$ Lynch syndrome $\cdot$ Gastritis $\cdot$ atrophic $\cdot$ Incidence

Electronic supplementary material The online version of this article (https://doi.org/10.1007/s10120-020-01113-0) contains supplementary material, which is available to authorized users.

Masayoshi Yamada

masyamad@ncc.go.jp

1 Endoscopy Division, National Cancer Center Hospital, 5-1-1, Tsukiji, Chuo-ku, Tokyo 104-0045, Japan

2 Division of Pathology and Clinical Laboratories, National Cancer Center Hospital, 5-1-1, Tsukiji, Chuo-ku, Tokyo 104-0045, Japan

3 Department of Genetic Medicine and Services, National Cancer Center Hospital, 5-1-1, Tsukiji, Chuo-ku, Tokyo 104-0045, Japan

4 Department of Clinical Genomics, National Cancer Center Research Institute, 5-1-1, Tsukiji, Chuo-ku, Tokyo 104-0045, Japan
5 Biostatistics Division, Center for Research Administration and Support, National Cancer Center, 5-1-1, Tsukiji, Chuo-ku, Tokyo 104-0045, Japan

6 Department of Gastric Surgery, National Cancer Center Hospital, 5-1-1, Tsukiji, Chuo-ku, Tokyo 104-0045, Japan

7 Oncogene Research Unit/Cancer Prevention Unit, Tochigi Cancer Center Research Institute, 5-1-1, Tsukiji, Chuo-ku, Tokyo 104-0045, Japan 


\section{Introduction}

Lynch syndrome (LS) is an inherited cancer predisposition syndrome caused by germline pathogenic variants in four mismatch repair (MMR) genes: $M L H 1, M S H 2, P M S 2$, and MSH6 [1]. This autosomal dominant disease is characterized by a high incidence of colorectal cancer $(60-80 \%)$ and endometrial cancer (30-70\%), which are frequently diagnosed at an early age $[2,3]$. LS patients also have elevated risks for tumors of other organs, including stomach, small bowel, gallbladder, biliary tract, pancreas, urothelial tract, ovary, and skin $[4,5]$.

Colorectal cancers in LS patients have several clinicopathological characteristics, including predominantly proximal localization, synchronous and metachronous occurrence of multiple tumors, and earlier onset [6]. LS-associated colorectal cancers tend to show histological features, such as prominent infiltrating lymphocytes, Crohn-like peritumoral lymphocytic reaction, poor differentiation, mucinous adenocarcinoma, signet-ring cell features, and a medullary growth pattern [7]. These clinicopathological features have been used to clinically screen patients with LS. In contrast, although gastric cancer is also known as one of the LSrelated tumors, the clinicopathological features of gastric cancer in LS patients are poorly understood.

Endoscopic surveillance for colorectal cancer has been shown to reduce the colorectal cancer-related mortality in patients with LS, and thus regular colonoscopy is strongly recommended $[8,9]$. However, the effectiveness of surveillance using upper gastrointestinal endoscopy in patients with LS remains controversial, and the recommendation varies among regions [10]. One of the reasons for the controversy is the geographical variation of gastric cancer incidence. As in sporadic cases, the cumulative incidence of gastric cancer in LS is high, up to $40 \%$, in some countries, including Japan and Korea [11-13], whereas it is low, ranging from 1 to $10 \%$, in western countries $[2,4,5,14,15]$. Based on these reports, we expect that environmental factors, particularly Helicobacter pylori (H. pylori) infection rates, would significantly affect gastric cancer risks in patients with LS. Some reports suggested that endoscopic surveillance of LS patients for gastric cancer should be provided based on the estimated risk in each patient $[3,16]$; however, limited data are available on the incidence of gastric neoplasms in patients with LS in Asian countries [11-13].

The present study aimed to determine the cumulative incidence and clinicopathological features of gastric neoplasms in patients with LS in Japan, where the incidence of gastric cancer is high in the general population.

\section{Materials and methods}

\section{Cases and data collection}

This study was approved by the Ethics Committee of the National Cancer Center, Tokyo, Japan (2013-011, 2013 303, and 2016-447). Cases were selected from the LS database in the National Cancer Center Hospital, Japan. Both probands and relatives are included in the database. All the patients were genetically confirmed to have a pathogenic MMR gene variant between September 1998 and June 2018 (Fig. 1). Inclusion criteria of this study were patients (1) who underwent upper gastrointestinal endoscopy at least once at our institution and/or (2) with a past history of gastric cancer. Clinical data until April 30, 2019 were included in the analysis.

\section{Genetic testing for germline MMR gene variants}

Genetic testing for germline MMR gene variants was performed by reverse transcription-polymerase chain reaction (RT-PCR) followed by direct sequencing analysis using puromycin-treated peripheral blood lymphocytes and/or direct sequencing and multiplex ligation-dependent probe amplification (MLPA) using genomic DNA obtained from peripheral blood lymphocytes, as previously described [17-19]. All pathogenic alternations are listed in Supplementary Table 1. MMR gene variants with frameshift mutations, nonsense mutations, or large deletion were regarded as pathogenic. For splice site variants, those previously reported in ClinVar or confirmed to show abnormal splicing as evaluated by RT-PCR were considered pathogenic. For missense variants, only those reported in ClinVar to be pathogenic were included. Constitutional hyper-methylation

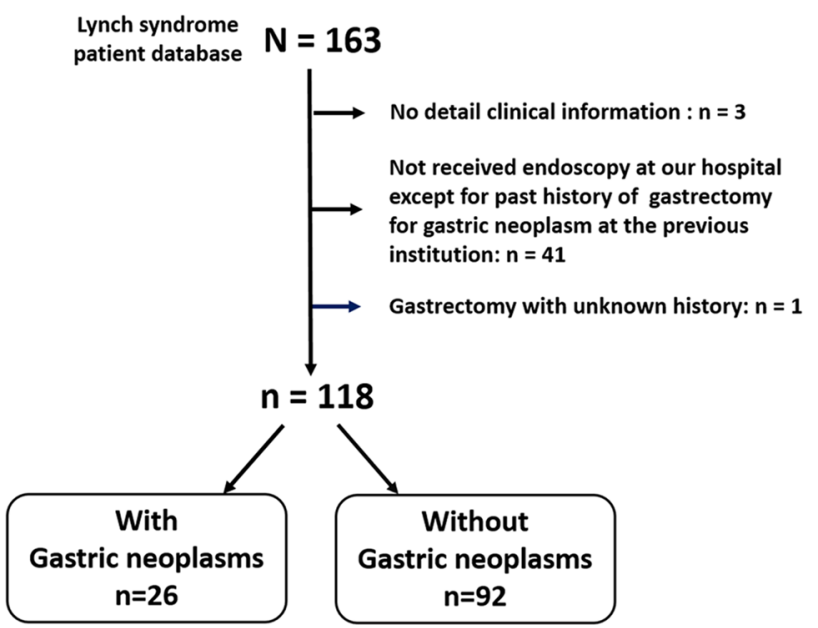

Fig. 1 Patient flowchart 
of the $M L H 1$ promoter region was detected by Na-bisulfite $\mathrm{PCR} /$ single-strand conformation polymorphism (SSCP), as previously described $[20,21]$.

\section{Clinicopathological findings}

The medical records of each patient were reviewed for clinical history, endoscopic findings, and histological findings of gastric neoplasms. Intramucosal neoplasm included dysplasia, which is defined as a non-invasive tumor, and intramucosal cancer, which invades the lamina propria or muscularis mucosae. The pathological diagnosis for gastric neoplasms was based on the WHO classification [1] and tumor staging was performed in accordance with the Union for International Cancer Control (UICC) TNM classification system. Information on lesions treated at other institutions was collected from referral letters. Gastric neoplasms detected at different time points were regarded as metachronous lesions. In the case of synchronous multiple lesions, TNM staging was performed for the lesion with the highest $\mathrm{T}$ stage. Endoscopic findings of gastric neoplasms were re-evaluated by two endoscopists (H.C. and M.Y.).

\section{Assessment of gastric atrophy}

The presence of gastric atrophy was evaluated by endoscopic images and/or histological findings of resection and biopsy specimens. Endoscopic evaluation of gastric atrophy was made based on Kimura-Takemoto classification [22]. Histologically, the presence of intestinal metaplasia was regarded as gastric atrophy [23]. The cases associated with atrophic change in either endoscopic or histological findings were deemed positive for gastric atrophy. Cases without appropriate endoscopic images and tissue samples were regarded as non-evaluable for gastric atrophy.

\section{Immunohistochemistry}

Immunohistochemical staining for MMR proteins was performed for all available specimens as described previously [24]. Deparaffinized 4- $\mu \mathrm{m}$-thick sections from each paraffin block were exposed to $0.3 \%$ hydrogen peroxide for $15 \mathrm{~min}$ to block endogenous peroxidase activity. Antigen retrieval was performed by autoclaving sections in $10 \mathrm{mM}$ citrate buffer (pH 6.0) for $10 \mathrm{~min}$. Sections were stained with primary antibodies including anti-MLH1 (ES05, 1:200 dilution; Dako, Glostrup, Denmark), anti-MSH2 (FE11, 1:200 dilution; Calbiochem, La Jolla, CA, USA), anti-PMS2 (A16-4, 1:200 dilution; Biocare Medical, Concord, CA, USA), and anti-MSH6 antibodies (SP93, 1:200 dilution; Spring Bioscience, Pleasanton, CA, USA). We used an automated stainer (Dako) and EnVision Detection System (Dako) according to the vendor's protocol. Non-neoplastic epithelial and stromal cells served as internal positive controls. Tumors showing significantly reduced or loss of expression of any MMR proteins were deemed to be MMR-deficient. The immunohistochemical staining results were evaluated by a single pathologist (S.S.).

\section{Microsatellite instability (MSI) testing}

MSI testing was performed with a Promega MSI Analysis System (Promega, Madison, WI, USA) for tumors that retained MMR protein expression, as previously described [25]. DNA was extracted following microdissection under a microscope of formalin-fixed, paraffin-embedded (FFPE) samples from both tumors and non-neoplastic areas.

\section{Statistical analysis}

Cumulative risks of gastric neoplasm were estimated using the Kaplan-Meier method, and log-rank test was used to compare risks among groups. Time to the occurrence of the gastric neoplasm was defined from birth to the age of gastric neoplasm occurrence or was censored at the date of the death, or date of the last contact, whichever was first. If patients developed multiple gastric neoplasms, only the age at first onset was counted for Kaplan-Meier analysis. Regarding the Kaplan-Meier analysis of invasive cancer, patients who underwent treatment at another institution and unavailable for detailed histology were excluded $(n=6)$. Patients not evaluated for gastric atrophy were excluded from Kaplan-Meier analysis, depending on atrophic status. A $P$ value $\leq 0.05$ was considered statistically significant. All statistical analyses were performed with BellCurve for Excel (Social Survey Research Information Co., Ltd., Tokyo, Japan).

\section{Results}

Of the 163 patients confirmed to have MMR gene pathogenic alteration, three patients without detailed clinical information, 41 without endoscopic examination at our institution, and one who received gastrectomy for unknown reason were excluded. Nine patients who underwent gastrectomy for gastric neoplasm before referral to our institution were included. Among the nine patients, detailed histology was available for three, but unknown for six patients. Finally, 118 individuals with LS of 79 pedigrees were included in the present study (Fig. 1).

\section{Characteristics of enrolled patients}

The baseline characteristics of the enrolled individuals with LS are shown in Table 1. Seventy five of these individuals 
Table 1 Baseline characteristics of Lynch syndrome carriers

\begin{tabular}{lcc}
\hline Baseline characteristics & Total $(n=118)$ & $\begin{array}{l}\text { Patients with gastric } \\
\text { neoplasm }(n=26)\end{array}$ \\
\hline Sex & & \\
Male & $52(44 \%)$ & 12 \\
Female & $66(56 \%)$ & 14 \\
MMR gene variant & & \\
MLH1 & $42(36 \%)$ & 10 \\
MSH2 & $66(56 \%)$ & 15 \\
MSH6 & $5(4 \%)$ & 1 \\
PMS2 & $5(4 \%)$ & 0 \\
Proband or relative & & \\
Proband & $75(64 \%)$ & 20 \\
Relative & $43(36 \%)$ & 6 \\
Amsterdam criteria II & & \\
Fulfilled & $89(75 \%)$ & 21 \\
Not fulfilled & $29(25 \%)$ & 5 \\
Family history ${ }^{\mathrm{b}}$ of gastric neoplasm & \\
Present & $79(67 \%)$ & 19 \\
Absent & $39(33 \%)$ & 7 \\
Gastric atrophy & & \\
Present & $54(46 \%)$ & 19 \\
Absent & $53(45 \%)$ & 0 \\
Not evaluable & $11(9 \%)$ & 7 \\
\hline
\end{tabular}

$M M R$ mismatch repair

${ }^{a}$ Lynch syndrome-associated tumors in Amsterdam criteria II include cancers of the colorectum, endometrium, stomach, small intestine, hepatobiliary, and urinary tract

${ }^{b}$ In first- and second-degree relatives

${ }^{\mathrm{c}}$ Gastric atrophy was evaluated endoscopically and/or histologically

were probands and 43 were relatives. We found that 89 (75\%) individuals from 56 pedigrees fulfilled the Amsterdam criteria II, whereas 77 (65\%) individuals from 47 pedigrees fulfilled the Amsterdam criteria II when gastric cancer was excluded from the LS-related tumors. The study patients were born between December 1919 and June 1995. The median age of the enrolled patients at the endpoint of study period; the mean age of occurrence of gastric neoplasm or censoring was 52 (range, 23-86) years old. A total of 26 (22.0\%) individuals from 22 pedigrees were diagnosed with gastric neoplasm (Supplementary Tables 1, 2). We found that 10,15 , and 1 patient developed gastric neoplasms among $42 \mathrm{MLH1}, 66 \mathrm{MSH}$, and $5 \mathrm{MSH} 6$ variant carriers, respectively. No gastric neoplasm was diagnosed among five $P M S 2$ variant carriers. Gastric atrophy was observed in 54 patients among 107 patients evaluable for endoscopic and/ or histological findings.

H. pylori infection status was tested in $48(41 \%)$ patients by urease breathing test or rapid urease test. All 16 individuals with $H$. pylori-positive result had gastric atrophy, whereas only 9 of $32 \mathrm{H}$. pylori-negative cases showed gastric
Table 2 Clinical characteristics of gastric neoplasms in Lynch syndrome patients $(n=58)$

\begin{tabular}{|c|c|}
\hline \multicolumn{2}{|l|}{ Clinical characteristics of gastric neoplasms } \\
\hline Number of lesions in one patient, median (range) & $1.5(1-11)$ \\
\hline Patients with a single lesion & 13 \\
\hline Patients with multiple lesions & 13 \\
\hline Synchronous & 4 \\
\hline Metachronous & 6 \\
\hline Synchronous and metachronous & 3 \\
\hline \multicolumn{2}{|l|}{ T stage ${ }^{\mathrm{a}}, n(\%)(n=58)$} \\
\hline Tis & $18(31)$ \\
\hline $\mathrm{T} 1$ & $27(47)$ \\
\hline $\mathrm{T} 2$ & $3(5)$ \\
\hline $\mathrm{T} 3$ or $\mathrm{T} 4$ & $4(7)$ \\
\hline Unknown $^{\mathrm{c}}$ & $6(10)$ \\
\hline \multicolumn{2}{|l|}{ TNM stage $^{\mathrm{b}}, n(\%)(\mathrm{n}=37)$} \\
\hline Stage 0 & $6(16)$ \\
\hline Stage I & $20(54)$ \\
\hline Stage II & $3(8)$ \\
\hline Stage III & $1(3)$ \\
\hline Stage IV & $1(3)$ \\
\hline Unknown $^{\mathrm{c}}$ & $6(16)$ \\
\hline
\end{tabular}

${ }^{\mathrm{a}} \mathrm{T}$ stage was classified according to UICC 8th edition for all lesions

${ }^{\mathrm{b}}$ TNM staging was classified according to UICC 8th edition. For synchronous multiple lesions, TNM staging was performed for the lesion with the highest $\mathrm{T}$ stage

${ }^{\mathrm{c}} \mathrm{T}$ stage and TNM stage were not available for six lesions that were previously treated in other institutions

atrophy. Thus, atrophic status was associated with $H$. pylori status in $39(81 \%)$ patients.

\section{Clinicopathological features of gastric neoplasms}

A total of 58 gastric neoplasms were detected in 26 patients (Table 2, Supplementary Fig. 1). This included 34 adenocarcinomas and 18 dysplasia cases, whereas 6 lesions treated in other institutions were not evaluable for histology. Notably, half of the LS patients with gastric neoplasms (13 patients, $50 \%$ ) developed synchronous and/or metachronous multiple gastric neoplasms. The initial diagnosis of gastric neoplasm was made at a median age of 54 (range, 39-81) years. In 37 gastric neoplasms that were assessed for pathological TNM stages, most were classified to early stages (stage 0/I) (26 lesions, $70 \%$ ). The median follow-up period after the resection of first gastric lesion was 10.3 (range, 0.4-30.0) years. Recurrence was observed in one patient, who died of the disease. Gastric lesion was the first neoplasm in two (8\%) patients (Supplementary Table 2).

Among the gastric neoplasms detected in patients with LS, 49 lesions in 19 patients were resected at our institution and eligible for detailed clinicopathological analysis 
(Supplementary Table 3). Twenty-four lesions were resected endoscopically and 25 lesions were surgically treated. Eighteen lesions (37\%) were dysplasia and 31 lesions $(63 \%)$ were diagnosed as adenocarcinoma. Histologically, most lesions were tubular or papillary adenocarcinoma (26 lesions, 84\%). Mixed-type (three lesions, $10 \%$ ) and poorly cohesive adenocarcinoma (two lesions, 6\%) were rare. We did not observe prominent intraepithelial lymphocytes or evident Crohn'slike reaction in any of the lesions.

Immunohistochemical staining showed that 42 lesions (91\%) were MMR-deficient and four (8\%) lesions retained MMR protein expression (Table 3, Fig. 2). All patients with gastric neoplasms retained MMR protein expression had at least one MMR-deficient gastric or colorectal neoplasm, suggesting that the germline MMR gene variants in these patients lead to loss of MMR protein expression. Immunohistochemical analysis was not performed in three lesions due to unavailability of tumor specimens or absence of informed consent for the immunohistochemical study.

\section{MSI testing on tumors with retained MMR protein expression}

We next explored MSI in the four gastric lesions with retained MMR protein expression. The DNA sample for one lesion, an intramucosal signet-ring cell carcinoma, was insufficient. Two of the remaining three lesions were MSS, whereas the other one was MSI-high. Details of the four lesions with retained MMR protein expression are shown in Supplementary Table 4.

\section{Cumulative risk of gastric neoplasm}

A Kaplan-Meier analysis demonstrated that the cumulative incidence ratios of developing gastric neoplasm, including dysplasia, in pathogenic MMR variant carriers were $41.0 \%$ [95\% confidence interval (CI), 26.9-55.0] at 70 years of age (Fig. 3a, Supplementary Table 5). The cumulative incidence ratio of gastric cancer to age 70 years was $27.0 \%$ (95\% CI, 14.3-39.6) (Fig. 3b). Males tended to develop gastric neoplasm at an earlier age compared with females (median age; male: 65 years vs. female: 75 years) (Fig. 4a, Supplementary Table 5). As to genetic variants, gastric neoplasms tended to occur in individuals with $M L H 1$ and $M S H 2$ variants (Fig. 4b), although the numbers of individuals with MSH6 and PMS2 variants were rather limited in our registry. Among 54 patients with gastric atrophy, 19 patients were diagnosed with gastric neoplasm, whereas no gastric neoplasm occurred in patients without gastric atrophy. The individuals with gastric atrophy had a significantly higher risk of developing gastric neoplasm compared with those without gastric atrophy $(P=0.026)($ Fig. $4 c)$. The Kaplan-Meier
Table 3 MMR protein expression in gastric neoplasms in Lynch syndrome patients $(n=49)$

\begin{tabular}{lccc}
\hline IHC staining result for MMR proteins & Total, $n(\%)$ & Dysplasia, $n(\%)$ & Cancer, $n(\%)$ \\
\hline Loss of any MMR protein & $42(86)$ & $14(78)$ & $28(90)$ \\
Retained MMR protein expression & $4(8)$ & $2(11)$ & $2(6)$ \\
Not available & $3(6)$ & $2(11)$ & $1(3)$ \\
\hline
\end{tabular}

$I H C$ immunohistochemical staining, $M M R$ mismatch repair

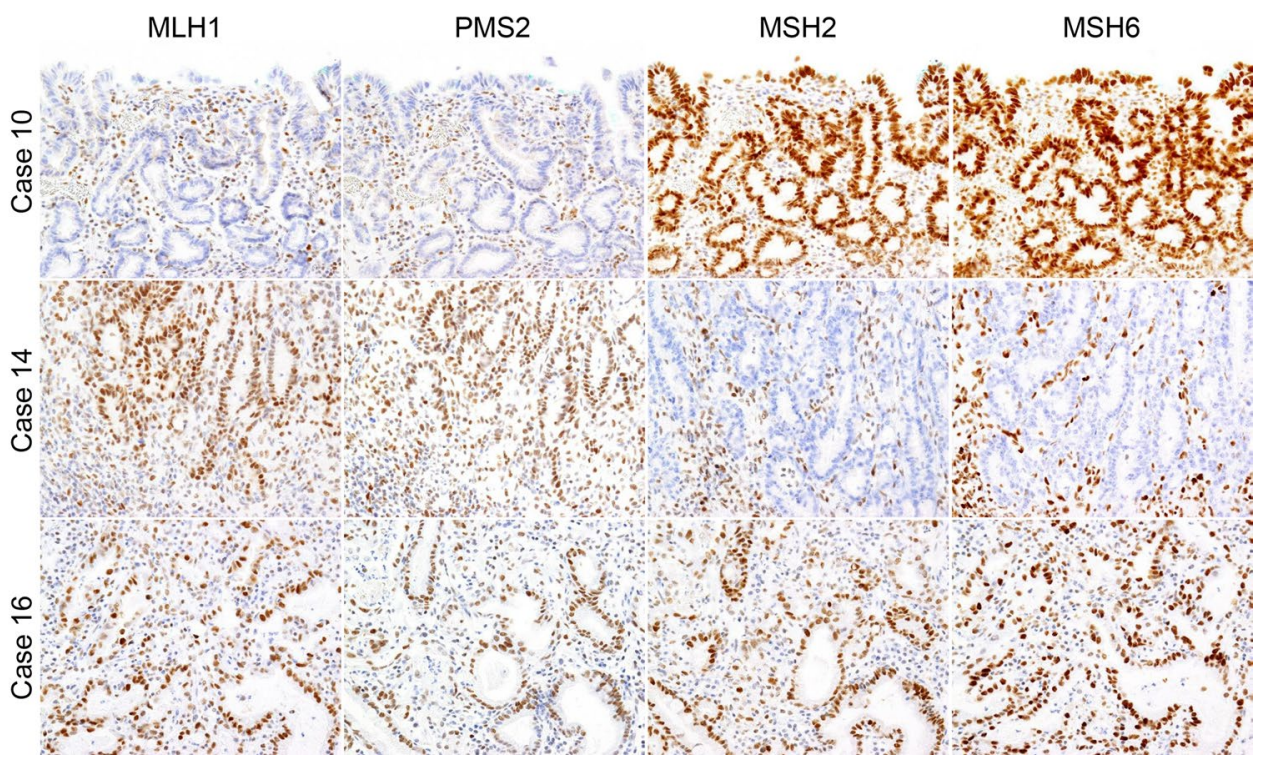

Fig. 2 Immunohistochemical staining for mismatch repair (MMR) proteins. (Case 10) Dysplasia in an $\mathrm{MLH1}$ variant carrier, showing loss of MLH1 and PMS2 and retention of MSH2 and MSH6. (Case 14) Advanced gastric cancer (T2) in an $\mathrm{MSH} 2$ variant carrier, showing loss of MSH2 and MSH6 and retention of MLH1 and PMS2. (Case 16) Intramucosal cancer (T1a) in an MSH2 variant carrier. Expressions of all four MMR proteins are retained 
Fig. 3 Cumulative incidence of gastric neoplasms in Lynch syndrome carriers. a Cumulative incidence of all gastric neoplasms, including dysplasia and cancers. b Cumulative incidence of invasive cancer. Patients with unknown detailed pathological results were excluded from the analysis $(n=6)$ a

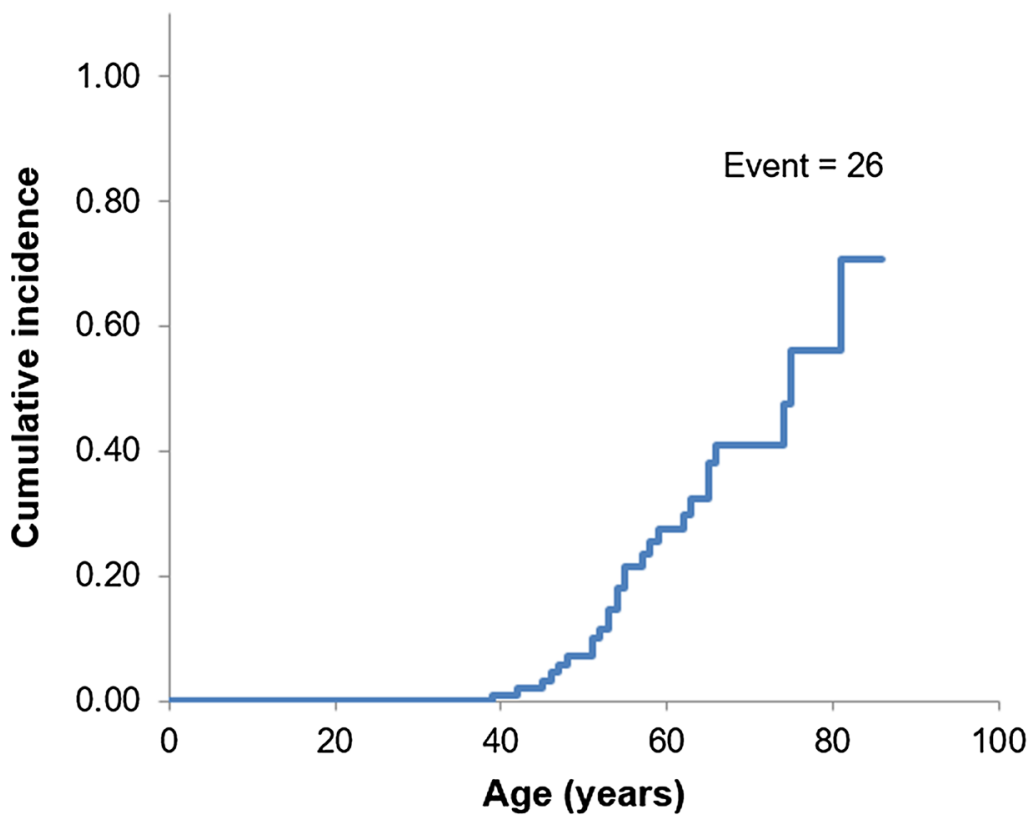

Number at risk 118

118

93

36

4

b

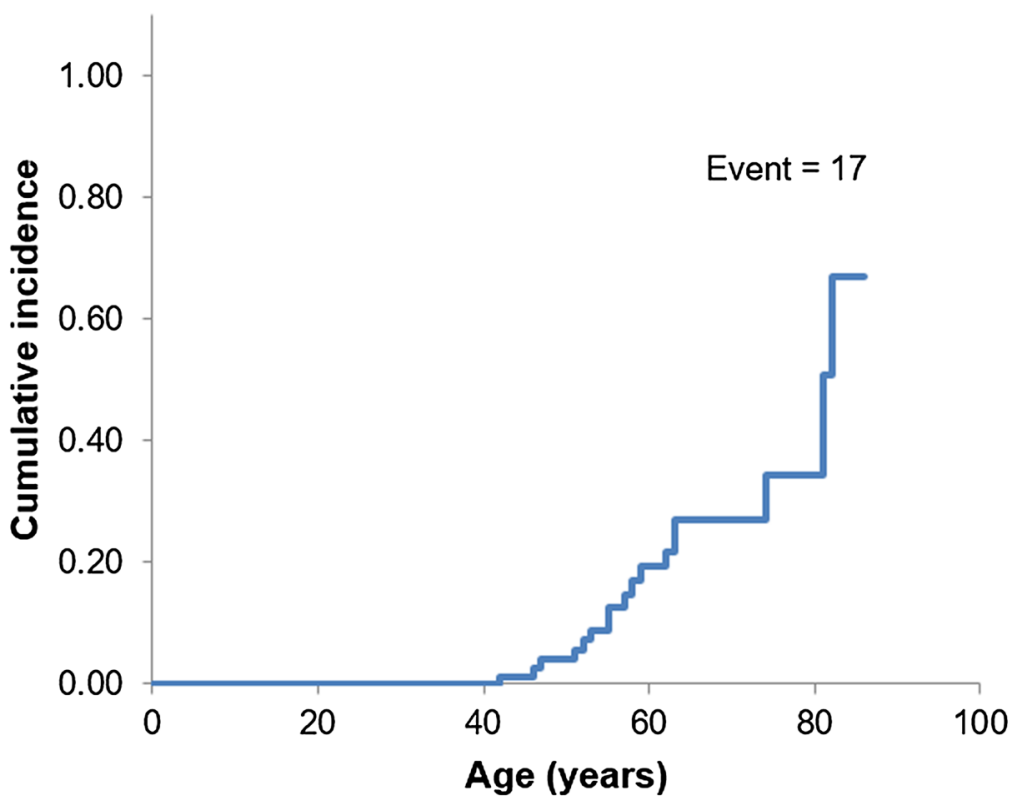

Number at risk 112

88

37

5 analysis revealed that the incidence ratios of gastric neoplasm in probands and relatives were not significantly different (Supplementary Fig. 2a). In total, six gastric neoplasms in 43 relatives were observed. The incidence risk of gastric neoplasms was not associated with the Amsterdam criteria II or family history of gastric neoplasm (Supplementary Figs. $2 b$ and 2c).

\section{Discussion}

Japan has a high incidence of gastric cancer, and the estimated lifetime incidence rate for gastric neoplasm in the general population reaches $11 \%$ in males and $5 \%$ in females [26]. However, the incidence risk of gastric neoplasms in LS patients estimated by our data was substantially higher 

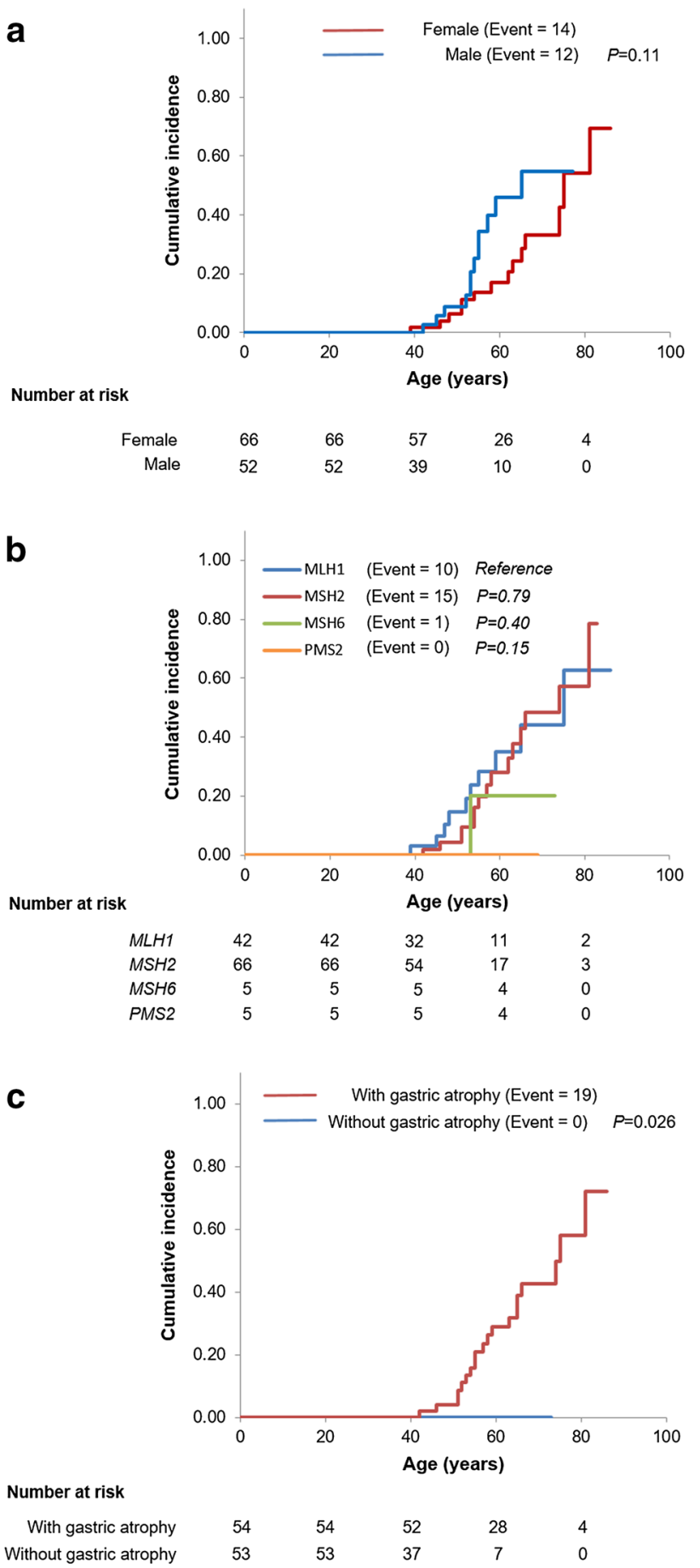

Fig. 4 Cumulative incidence of gastric neoplasms depending on (a) sex, (b) MMR gene variants, and (c) endoscopically and/or histologically evaluated gastric atrophy. In (b), $P$ values reflect comparisons between each MMR gene and $M L H 1$ variant

compared with the general population risk, indicating that LS is a risk factor for gastric cancer. Previous studies in gastric cancer in LS patients in western countries reported a 1-10\% life-time incidence of gastric cancer $[2,4,5,14$,
15]. These results suggest that the geographical difference in gastric cancer risk also applies to patients with LS.

Currently, two studies have examined the gastric cancer incidence in LS patients with proven MMR gene variants from Asian countries. Saita et al. reported that 10 out of 55 patients with LS developed gastric cancer, and the cumulative gastric cancer risk of these patients was about $14.5 \%$ at the age of 70 [13]. Ikenoue et al. reported that 10 out of 64 LS patients from a multi-center registration study had a history of gastric cancer, and the cumulative risk of gastric cancer was estimated about 39\% at the age of 70 [11]. These results were consistent with the present study, which reported a higher gastric cancer incidence compared with the incidence in western countries.

Notably, cases with gastric neoplasms in this study invariably showed gastric atrophy. The Kaplan-Meier analysis demonstrated that gastric neoplasms occurred significantly more frequently in individuals with gastric atrophy. The vast majority of sporadic gastric cancers in Japan are related to chronic atrophic gastritis due to $H$. pylori infection [27]. H. pylori was frequently positive in patients with gastric atrophy in our study. We speculate that $H$. pylori infection also plays a major role in the development of gastric cancer in patients with LS. Furthermore, the regional difference in gastric cancer incidence in LS carriers might be explained by the differential $H$. pylori infection rates. Therefore, $H$. pylori eradication to prevent gastric cancer may be suggested in LS patients with a history of $H$. pylori infection.

Half of the patients with gastric neoplasms in the present study developed synchronous or metachronous multiple gastric tumors. Although the risk of multiple tumor occurrence is a well-recognized feature of LS-associated colorectal cancer [6], to the best of our knowledge, the present study is the first to describe the frequent occurrence of multiple gastric neoplasms in LS.

Most of the gastric neoplasms in LS patients showed loss of MMR proteins consistent with the germline variant, supporting the causative role of MMR gene variants in these lesions. In agreement with our observation, Saita et al. recently reported the loss of MMR proteins in all eight gastric cancers examined immunohistochemically in the study [13]. Notably, our study showed that loss of MMR protein expression was also frequent in dysplasia. This suggests that the acquisition of MMR deficiency occurs in the early stage of gastric tumorigenesis associated with LS, similar to colorectal cancer [24]. However, two dysplasia and two adenocarcinomas retained MMR protein expression in our series. Among these, two lesions showed MSS. This indicates that MMR-proficient "sporadic" gastric cancer can also occur in LS patients. Indeed, there appears to be a difference in the contribution of MMR deficiency among tumors of LS patients depending on the organ of their origin. Although virtually all colorectal cancers in patients with LS exhibit 
MMR deficiency, half of LS-associated breast cancers are MMR-proficient [28]. Our analysis suggests that MMR deficiency is involved in the development of most but not all LS-associated gastric cancers.

Notably, the majority of LS-associated gastric cancers were intestinal type in Lauren classification [29], including tubular and papillary adenocarcinoma. Consistently, Aarnio et al. reported that the intestinal type was predominant, accounting for $79 \%$ of gastric cancers in LS patients, including proven MMR variant carriers and those that fulfilled the Amsterdam criteria [30]. Previous studies showed sporadic gastric cancers with microsatellite instable subtype, related to hypermethylation of the $M L H 1$ promoter [31], were also predominantly intestinal-type [31,32]. Regardless of the difference in genetic background, the intestinal-type histology may be a common characteristic of MSI-high gastric cancer.

Some potential limitations exist in this study. First, there might be a bias of study population. Recently, universal tumor testing for LS revealed that MSH6 and PMS2 carriers are more frequent than previously recognized [33]. In contrast, most of the registered LS patients in our study were $M L H 1$ and $M S H 2$ carriers, which are potentially at high risk of LS-associated cancers [4]. Thus, we cannot exclude the possibility of overestimation of gastric cancer incidence compared with the genuine LS population, including asymptomatic carriers. Second, information on $H$. pylori status was not available for all patients. However, both endoscopic findings of gastric atrophy [34] and histological assessment of intestinal metaplasia [23] were reported to be an accurate alternative for atrophic gastritis with excellent interobserver agreement. As atrophic gastritis has been reported to be strongly associated with $H$. pylori in Japan [35], we consider that the endoscopic and histological findings can be a good substitute for $H$. pylori status in clinical settings.

In conclusion, the present study demonstrated the high incidence of gastric neoplasms in LS patients in Japan. Patients with LS, particularly those with atrophic gastritis, are at high risk of gastric dysplasia and cancer, and the occurrence of synchronous and/or metachronous multiple lesions is common. Our results strongly suggest the requirement for endoscopic surveillance for LS patients, especially for those with atrophic gastritis in countries with a high incidence of gastric cancer.

Acknowledgements We thank Ms. Sachiko Miura, Ms. Toshiko Sakaguchi and Ms. Chizu Kina for their skillful technical assistance. This work was supported by The National Cancer Center Research and Development Fund (25-A-1, 31-A-2) and AMED under Grant Number JP19ck0106268h0003. We thank Edanz Group (https://en-author-servi ces.edanzgroup.com/) for editing a draft of this manuscript.

Author contributions $\mathrm{HC}$ analyzed data. MY contributed to study concept and design. MY, NT, MH, TY, TY, YS, IO, and KS provided clinical information. SS performed histological analysis. MU, MG, and $\mathrm{KS}$ carried out experiments. GO and AK supervised the statistical analysis. HC, MY, and SS wrote the manuscript. All authors contributed to discussions and had final approval of the submitted manuscript.

\section{Compliance with ethical standards}

Conflict of interest The authors declare that they have no conflict of interest.

Ethical approval All procedures involving human participants were performed in accordance with the ethical standards of the institutional and national research committee and with the Helsinki Declaration of 1964 and later versions. This study was approved by the Ethics Committee of the National Cancer Center, Tokyo, Japan (2013-011, 2013-303, and 2016-447).

\section{References}

1. Carneiro FFM, Grabsch HI, Yasui W. Gastric adenocarcinoma. In: Digestive system tumours. 5th edition: WHO Classification of Tumours Editorial Board. International research agency on cancer, 2019. p. 85-95.

2. Barrow E, Robinson L, Alduaij W, Shenton A, Clancy T, Lalloo $\mathrm{F}$, et al. Cumulative lifetime incidence of extracolonic cancers in Lynch syndrome: a report of families with proven mutations. Clin Genet. 2009;75(2):141-9.

3. Giardiello FM, Allen JI, Axilbund JE, Boland CR, Burke CA, Burt RW, et al. Guidelines on genetic evaluation and management of Lynch syndrome: a consensus statement by the US Multisociety Task Force on colorectal cancer. Am J Gastroenterol. 2014;109(8):1159-79. https://doi.org/10.1038/ajg.2014.186.

4. Bonadona V, Bonaiti B, Olschwang S, Grandjouan S, Huiart L, Longy $\mathrm{M}$, et al. Cancer risks associated with germline mutations in MLH1, MSH2, and MSH6 genes in Lynch syndrome. JAMA. 2011;305(22):2304-10. https://doi.org/10.1001/jama.2011.743.

5. Watson P, Vasen HFA, Mecklin JP, Bernstein I, Aarnio M, Jarvinen HJ, et al. The risk of extra-colonic, extra-endometrial cancer in the Lynch syndrome. Int J Cancer. 2008;123(2):444-9. https://doi.org/10.1002/ijc.23508.

6. Lynch HT, Lynch JF, Lynch PM, Attard T. Hereditary colorectal cancer syndromes: molecular genetics, genetic counseling, diagnosis and management. Fam Cancer. 2008;7(1):27-39.

7. Shia J, Holck S, DePetris G, Greenson JK, Klimstra DS. Lynch syndrome-associated neoplasms: a discussion on histopathology and immunohistochemistry. Fam Cancer. 2013;12(2):241-60.

8. Dove-Edwin I, Sasieni P, Adams J, Thomas HJ. Prevention of colorectal cancer by colonoscopic surveillance in individuals with a family history of colorectal cancer: 16 year, prospective, followup study. BMJ. 2005;331(7524):1047.

9. Järvinen HJ, Aarnio M, Mustonen H, Aktan-Collan K, Aaltonen LA, Peltomäki P, et al. Controlled 15-year trial on screening for colorectal cancer in families with hereditary nonpolyposis colorectal cancer. Gastroenterology. 2000;118(5):829-34.

10. Pan JY, Haile RW, Templeton A, Macrae F, Qin F, Sundaram V, et al. Worldwide practice patterns in Lynch syndrome diagnosis and management, based on data from the international mismatch repair consortium. Clin Gastroenterol Hepatol. 2018;16(12):190110. https://doi.org/10.1016/j.cgh.2018.04.025.

11. Ikenoue T, Arai M, Ishioka C, Iwama T, Kaneko S, Matsubara $\mathrm{N}$, et al. Importance of gastric cancer for the diagnosis and surveillance of Japanese Lynch syndrome patients. J Hum Genet. 2019;64(12):1187-94. 
12. Park YJ, Shin KH, Park JG. Risk of gastric cancer in hereditary nonpolyposis colorectal cancer in Korea. Clin Cancer Res. 2000;6(8):2994-8.

13. Saita C, Yamaguchi T, Horiguchi SI, Yamada R, Takao M, Iijima T, et al. Tumor development in Japanese patients with Lynch syndrome. PLoS ONE. 2018;13(4):e0195572. https://doi. org/10.1371/journal.pone.0195572.

14. Capelle LG, Van Grieken NC, Lingsma HF, Steyerberg EW, Klokman WJ, Bruno MJ, et al. Risk and epidemiological time trends of gastric cancer in Lynch syndrome carriers in the Netherlands. Gastroenterology. 2010;138(2):487-92. https://doi.org/10.1053/j. gastro.2009.10.051.

15. Engel C, Loeffler M, Steinke V, Rahner N, Holinski-Feder E, Dietmaier $\mathrm{W}$, et al. Risks of less common cancers in proven mutation carriers with lynch syndrome. J Clin Oncol. 2012;30(35):4409-15.

16. Vasen HF, Blanco I, Aktan-Collan K, Gopie JP, Alonso A, Aretz $\mathrm{S}$, et al. Revised guidelines for the clinical management of Lynch syndrome (HNPCC): recommendations by a group of European experts. Gut. 2013;62(6):812-23. https://doi.org/10.1136/gutjn 1-2012-304356.

17. Nomura S, Sugano K, Kashiwabara H, Taniguchi T, Fukayama N, Fujita S, et al. Enhanced detection of deleterious and other germline mutations of hMSH2 and hMLH1 in Japanese hereditary nonpolyposis colorectal cancer kindreds. Biochem Biophys Res Commun. 2000;271(1):120-9.

18. Sugano K, Nakajima T, Sekine S, Taniguchi H, Saito S, Takahashi M, et al. Germline PMS2 mutation screened by mismatch repair protein immunohistochemistry of colorectal cancer in Japan. Cancer Sci. 2016;107(11):1677-86.

19. Adachi M, Banno K, Masuda K, Yanokura M, Iijima M, Takeda $\mathrm{T}$, et al. Carcinoma of the lower uterine segment diagnosed with Lynch syndrome based on MSH6 germline mutation: a case report. J Obstet Gynaecol Res. 2017;43(2):416-20.

20. Miyakura Y, Sugano K, Konishi F, Ichikawa A, Maekawa M, Shitoh K, et al. Extensive methylation of hMLH1 promoter region predominates in proximal colon cancer with microsatellite instability. Gastroenterology. 2001;121(6):1300-9.

21. Miyakura Y, Sugano K, Akasu T, Yoshida T, Maekawa M, Saitoh $\mathrm{S}$, et al. Extensive but hemiallelic methylation of the hMLH1 promoter region in early-onset sporadic colon cancers with microsatellite instability. Clin Gastroenterol Hepatol. 2004;2(2):147-56.

22. Kimura K, Takemoto T. An endoscopic recognition of the atrophic border and its significance in chronic gastritis. Endoscopy. 1969;1(03):87-97.

23. Capelle LG, de Vries AC, Haringsma J, Ter Borg F, de Vries RA, Bruno MJ, et al. The staging of gastritis with the OLGA system by using intestinal metaplasia as an accurate alternative for atrophic gastritis. Gastrointest Endosc. 2010;71(7):1150-8.

24. Sekine S, Mori T, Ogawa R, Tanaka M, Yoshida H, Taniguchi $\mathrm{H}$, et al. Mismatch repair deficiency commonly precedes adenoma formation in Lynch Syndrome-associated colorectal tumorigenesis. Mod Pathol. 2017;30(8):1144-51. https://doi. org/10.1038/modpathol.2017.39.

25. Bando H, Okamoto W, Fukui T, Yamanaka T, Akagi K, Yoshino $\mathrm{T}$. Utility of the quasi-monomorphic variation range in unresectable metastatic colorectal cancer patients. Cancer Sci. 2018;109(11):3411-5.

26. Cancer registry and statistics. Cancer information service, National Cancer Center, Japan. 2018. https://ganjoho.jp/en/profe ssional/statistics/table_download.html.

27. Matsuo T, Ito M, Takata S, Tanaka S, Yoshihara M, Chayama $\mathrm{K}$. Low prevalence of helicobacter pylori-negative gastric cancer among Japanese. Helicobacter. 2011;16(6):415-9.

28. Walsh MD, Buchanan DD, Cummings MC, Pearson S-A, Arnold ST, Clendenning M, et al. Lynch syndrome-associated breast cancers: clinicopathologic characteristics of a case series from the colon cancer family registry. Clin Cancer Res. 2010;16(7):2214-24.

29. Lauren P. The two histological main types of gastric carcinoma: diffuse and so-called intestinal-type carcinoma: an attempt at a histo-clinical classification. Acta Pathol Microbiol Scand. 1965;64(1):31-49.

30. Aarnio M, Salovaara R, Aaltonen LA, Mecklin JP, Jarvinen HJ. Features of gastric cancer in hereditary non-polyposis colorectal cancer syndrome. Int J Cancer. 1997;74(5):551-5. https:// doi.org/10.1002/(sici)1097-0215(19971021)74:5<551:aid-ijc13 $>3.0 . c 0 ; 2-9$.

31. Wu MS, Lee CW, Shun CT, Wang HP, Lee WJ, Chang MC, et al. Distinct clinicopathologic and genetic profiles in sporadic gastric cancer with different mutator phenotypes. Genes Chromosomes Cancer. 2000;27(4):403-11.

32. Polom K, Marano L, Marrelli D, De Luca R, Roviello G, Savelli $\mathrm{V}$, et al. Meta-analysis of microsatellite instability in relation to clinicopathological characteristics and overall survival in gastric cancer. Br J Surg. 2018;105(3):159-67.

33. Espenschied CR, LaDuca H, Li S, McFarland R, Gau C-L, Hampel $\mathrm{H}$. Multigene panel testing provides a new perspective on Lynch syndrome. J Clin Oncol. 2017;35(22):2568-75.

34. Watanabe K, Nagata N, Nakashima R, Furuhata E, Shimbo T, Kobayakawa M, et al. Predictive findings for helicobacter pyloriuninfected,-infected and-eradicated gastric mucosa: validation study. World J Gastroenterol. 2013;19(27):4374.

35. Asaka M, Sugiyama T, Nobuta A, Kato M, Takeda H, Graham DY. Atrophic gastritis and intestinal metaplasia in Japan: results of a large multicenter study. Helicobacter. 2001;6(4):294-9.

Publisher's Note Springer Nature remains neutral with regard to jurisdictional claims in published maps and institutional affiliations. 